\title{
Estimation de la précision des campagnes acoustiques au Sénégal par la méthode géostatistique transitive à une dimension
}

\author{
Birane Samb (1) et Pierre Petitgas \\ (1) Centre de Recherches Océanographiques de Dakar-Thiuroye, BP 2241, Dakar, Sénégal. \\ E-mail: samb@isra.isra.sn \\ (2) ORSTOM, Centre de Montpellier, BP 5045, 34032 Montpellier Cedex 1, France. \\ E-mail:prititgas@orstom.rio.net
}

Reçu le 30 août 1996; accepté le 4 novembre 1996.

Samb B., P. Petityas, Aquat. Living Resour., 1997, 10, 75-82.

Use of the geostatistical one-dimensional transitive method in analysing Senegalese acoustic surveys.

\begin{abstract}
This study presents an application of the geostatistical one-dimensional (ID) transitive method for estimating the precision of acoustic pelagic abundance estimates in Senegal. In applying the method, the major problem encountered was how to take into account the temporal variability in the computation of the estimation variance. In acoustic surveys, echointegration is performed continuously along the ship's sailing track. For a survey design made of parallel transects, each transect can thus be considered as a sampling unit. By summing the density values along the transects to obtain biomass values per transect, the $2 \mathrm{D}$ estimation is simply reduced to a $1 \mathrm{D}$ estimation procedure. If the transects are equidistant, the geostatistical transitive method applied in 1D on the biomass values per transect gives a correct and simple solution to the estimation of the survey precision. The method has been applied to the Senegalese acoustic surveys which were performed with the same sampling design made of regularly spaced parallel transects oriented East-West.

The densities were summed along the transects. The latitudinal variation of the biomass per transect was presented as a 1D profile. Biomass, spatial distribution and its structure (autocovariance) showed great differences between surveys. Profiles were grouped in 4 categories which presented different structural characteristics. In each category, the average structure was estimated and modelled and the average relative estimation error was estimated. The relative estimation error varied with the differences in structure between categories but also with model hypothesis about the structure at small distance. Two hypothesis were considered, continuity of the distribution at small distance (absence of nugget effect), or discontinuity (presence of nugget effect). The maximum relative error estimated was $31 \%$. The major problem in modelling the structure of the $1 \mathrm{D}$ profile was to fit or not to fit a nugget effect. The interpretation of such nugget could be temporal variability at the scale of the transect.
\end{abstract}

Keywords: Geostatistics, variance, acoustics, abundance.

Résumé

L'étude présente une application de la méthode géostatistique transitive à une dimension (1D) pour estimer la précision des évaluations de biomasse effectuées au Sénégal par écho-intégration. Le problème majeur rencontré dans cette étude a été la prise en compte de la variabilité temporclle dans l'estimation de la variance d'échantillonnage. Lors des campagnes acoustiques, l'écho-intégration se fait en continu le long de la route du navire. Lorsque le plan d'échantillonnage est constitué de transects parallèles, cette caractéristique permet de considérer chaque transect comme une unité d'échantillonnage. En faisant la somme des valeurs mesurées le long des transects, l'estimation à $2 \mathrm{D}$ est simplement ramenéc à une estimation à $1 \mathrm{D}$. Si de plus les transects sont équidistants, la méthode géostatistique transitive à $1 \mathrm{D}$ offre une solution correcte et simple au calcul de la variance d'estimation. La méthode a été appliquée à la série 
des campagnes acoustiques sénégalaises qui ont toutes été effectuées sclon le même réseau de transects équidistants et parallèles d'orientation Est-Ouest.

Les densités sont sommées le long des transects. Les variations latitudinales de la biomasse par transect sont présentées sous forme de profils à 1D. La biomassc, la répartition spatiale ct l'autucorrélation sur ces profils présentent des différences importantes d'une campagne à l'autre. Les profils ont été regroupés en 4 catćgories présentant des caractéristiques différentes. L'autocorrćlation moyenne (structure) par catégorie a été estiméce et modélisée. Une valeur moyenne de l'erreur relative d'estimation est fournic pour chaque catégoric. L'crreur relative d'estimation varie en fonction des différences de structures entre catégories et aussi en fonction de différentes hypothèses pour la modélisation de la structure aux petites distances. Deux cas ont été considérés: continuité de la distribution (absence d'un effet pépite) ou discontinuité (présence d'un effet pépite). L'crreur relative maximale cstimée est d'environ $31 \%$. Le problème majeur rencontré à la modélisation de la structure ID des biomasses par transect a été de choisir de prendre en compte ou non un elfé de pépite. L'interprétation de cette pépite scrait la variabilité temporelle à l'échelle de la radiale.

Mots-clés : Gćostatistique, variance, acoustique, abondance.

\section{INTRODUCTION}

Parmi les ressources marines exploitées au Sénégal, les poissons pélagiques côtiers sont quantitativement les plus importants. Ils peuvent représenter les $2 / 3$ des captures des pêcheries artisanales et industrielles (Samba, 1994). La biomasse des espèces pélagiques côtières (principalement Clupéidés et Carangidés) fait l'objet d'une évaluation directe par écho-intégration, sur unc base annuelle depuis 1984. On cherche ici à cstimer la précision d'échantillonnage sur l'estimatcur de biomasse.

Les évaluations ont été effectuées à l'aide du N/O "Louis Sauger" du Sénégal avec le même matériel d'écho-intégration de type Biosonics et le même plan d'échantillonnage constitué de transects parallèles et équidistants. Dans ce type d'échantillonnage, les points échantillons étant prélevés en série, on ne peut estimer la précision d'échantillonnage sur l'estimation de la biomasse que si on spécilie les corrélations spatiales dans la population (Matheron, 1971; Cochran, 1977). $\mathrm{L}$ a géostatistique répond à ce problème en faisant l'hypothèse que le modèle de corrélation spatiale inféré caractérise la corrélation dans la population.

On a utilisé la méthode géostatistique transitive car elle est adaptéc au plan d'échantillonnage pratiqué et qu'elle présente un cas de simplification pour les campagnes acoustiques. Elle a été développée par Matheron (1971) pour estimer la quantité totale d'une variable sur une zone el sa variance d'estimation à partir d'un échantillonnage à maille régulière, dont l'origine est aléatoire (plan systćmatique de Cochran, 1977). Dans le cas des campagnes acoustiques, l'échantillonnage est effectué en continu le long de la route du navire. Cette propriété permet de considérer chaque transect comme une unité d'échantillonnage. En faisant la somme de toutes les densités élémentaires sur le transect, on ramène l'estimation de la quantité totale à un problème à une dimension (Jolly et Hampton, 1990; Petitgas, 1993). Pour estimer la variance d'estimation dans le cas d'échantillonnages par transects parallèles et équidistants, il a été proposé d'utiliser la méthode transitive à une dimension (1D) sur les valcurs cumulées par transect (Petitgas, 1993).
La présente étude constitue une application de la méthode dans laquelle on verra que le paramètre structural influençant le plus la variance d'estimation est la pépite à une dimension dont on donne une interprélation en terme de variabilité temporelle.

Différentes stratégies d'échantillonnage ont été comparées pour les campagnes d'écho-intégration lors de réunions de travail du Conseil International pour l'Exploration de la Mer - CIEM (Simmonds et al., 1992). Ces études ont porté sur l'échantillonnage spatial uniquement. Nous proposons ici d'utiliser un paramètre, "la pépite à $1 D$ », pour quantifier la variabilité temporelle présente dans les données des campagnes acoustiques.

\section{MATÉRIELS ET MÉTHODES}

\section{Couverture géographique}

Les données proviennent des campagnes océanographiques de prospection du programme ECHOSAR dont J'objectif principal est l'évaluation de l'abondance et la répartition des poissons pélagiques côtiers du plateau continental du Sénégal. Les biomasses mesurées constituent une fraction des populations de Clupéidés et Carangidés situćes dans la zone de balancement du "front thermique nord » qui oscille entre $10^{\circ} \mathrm{N}$ (îles Bissagos) et $21^{\circ} \mathrm{N}$ (cap Blanc).

Dans cette étude, nous nous sommes intéressés aux échantillonnages effectués du cap Vert $\left(14^{\circ} 45^{\prime} \mathrm{N}\right)$ au cap Roxo ( $\left.12^{\circ} 20^{\prime} \mathrm{N}\right)$, sur la côte sud du Sénégal. Sur cette zone, l'échantillonnage a été effectué le long de transects orientés Est-Ouest, entre 10 et $200 \mathrm{~m}$ de profondeur. La forme du plateau continental a déterminé la longueur des transects. La distance intertransect était de 5 milles nautiques. La couverture de lá zone correspond à 944 milles que le $\mathrm{N} / \mathrm{O}$ « Louis Sauger » a parcouru sans faire de double couverture jour/nuit, à une vitesse moyenne de 9 noeuds. Le même réscau de transects a été prospecté lors de chaque campagne océanographique. 
Seules les campagnes de février à avril (saison froide) ont été utilisées. Pendant cette période, les populations de petits pélagiques, qui effectuent des migrations du Nord vers le Sud puis du Sud vers le Nord, en début et fin de saison froide, sont considérés comme occupant principalement le plateau continental du Sénégal (Boely et al., 1982). Le Tableau 1 résume les caractéristiques des 8 campagnes étudiées de 1983 à 1993 à l'exception des années 1990 et 1991 durant lesquelles les prospections n'ont pas cu lieu.

\section{Écho-intégration}

Toutes les campagnes ont été réalisées avec le même navire, le même plan d'échantillonnage et le même matériel acoustique. L'échosondeur est de type Biosonics modèle 101. Sa fréquence nominale est de $120 \mathrm{kHz}$. Le transducteur est circulaire et émet dans un angle théorique de $10^{\circ}$ entre des points $-3 \mathrm{~dB}$ du diagramme de directivité. L'intégrateur numérique est de type Biosonics modèle 120 . Il est couplé au sondeur et relié à un ordinateur pour le stockage des données. L'échographe est de type Ross modèle Fine Line $250 \mathrm{~m}$ modifié par Biosonics.

ll est à noter que d'année en année, l'étalonnage effcctué par hydrophone a montré une très bonne stabilité du matériel, les mesures demeurant proches. La durée d'impulsion a été de $0,6 \mathrm{~ms}$. La distance élémentaire d'échantillonnage (ESDU) a été fixée à 1 mille nautique. L'écho du fond a été suivi manuellement pour éviter que l'écho de bancs denses, proches du fond ne soit interprété comme celui du fond. Le seuil d'intégration a été choisi par un même utilisateur, en début de campagne, et a varié d'une campagne à l'autre dans la gamme $80-120 \mathrm{mV}$, en fonction du bruit, du plancton et des gains à l'émission et à la réception.

L'écho-intégration a été effectuée par couches (Johannesson et Mitson, 1984). Une seule et même valeur d'index de reflexion (TS) moyenne $\left(-35,4 \mathrm{~dB} \cdot \mathrm{kg}^{-1}\right)$ a été utilisée pour toutes les campagnes. Cette valeur est basée sur des mesures en cage et sur les tailles moyennes débarquées par la pêcherie (Levenez, 1990). La densité moyenne en poissons par ESDU est exprimée en $\mathrm{kg} \cdot \mathrm{m}^{-2}$ de surface d'eau de mer. La biomasse des poissons pélagiques côtiers est évaluée sans distinction d'cspèce.

\section{Méthode géostatistique transitive à une dimension}

Les principes et les détails de la méthode transitive sont décrits par Matheron (1971) et Petitgas (1993). Dans la méthode transitive, les données ne sont pas interprétées comme une réalisation d'une fonction aléatoire. C'est le caractère aléatoire de l'origine de la grille d'échantillonnage qui permet de développer la formule de la variance d'estimation. L'outil structural est le covariogramme transitif. La méthode suppose que l'aire de distribution de la ressource a été entièrement couverte par l'échantillonnage.

Soient $z(x, y)$ la densité au point de longitude $x$ et de latitude $y, q(y)$ la quantité en poisson sur le transect d'ordonné y et $\mathrm{Q}$ la quantité totale à estimer. En dehors de la zone de présence du poisson $z(x, y)$ et $q(y)$ sont nulles. Pour des transects parallèles orientés Est-Ouest, Q s'écrit:

$$
\mathrm{Q}=\int_{-\infty}^{+\infty} d y \int_{-\infty}^{+\infty} z(x, y) d x=\int_{-\infty}^{+\infty} q(y) d y
$$

Soit $y_{0}$ la position du transect origine et $d$ la distance inter-transect, l'estimateur Q* de Q s'écrit:

$$
\mathrm{Q}^{*}\left(y_{0}\right)=d \sum_{k=-x}^{+\infty} q\left(y_{0}+k d\right)
$$

La variance d'estimation est (Matheron, 1971):

$$
\sigma_{Q^{2}}=d \sum_{k=-\infty}^{+\infty} g_{1 D}(k i d)-\int_{-\infty}^{+\infty} g_{1 D}(h) d h
$$

avec $g_{1 D}(h)=\int_{-\infty}^{+\infty} q(y) q(y+h) d y, g_{1 D}(h)$ est le covariogramme transitif de la biomasse par transect

\begin{tabular}{|c|c|c|c|}
\hline Campagne & Date & $Q^{*}$ (tonnes) & Documents internes du CRODT* \\
\hline Echosar 8 & $10-18$ avril 1985 & 744051 & Levenez $(1996 a)$, sous presse \\
\hline Echosar 12 & 12 février-1 $^{\text {er }}$ mars 1987 & 338518 & Samb (1988a), archive $n^{\circ} 168$ \\
\hline Echosar 14 & 13-24 février 1988 & 463333 & Samb (1988b), archive $\mathrm{n}^{\circ} 172$ \\
\hline Echosar 15 & $7-19$ mars 1989 & 86374 & Samb et Levenez (1995), archive $\mathrm{n}^{\circ} 184$ \\
\hline
\end{tabular}

Tableau 1. - Caractéristiques des campagnes Echosar utilisées. Q* représente la valcur estimée de la biomasse pélagique sur la côte sud du Sénégal. Characteristics of Echosar survey used. Q* represents the estimated value of the pelagic biomass for the southern coast of Senegal.

* CRODT : Centre de Recherches Océanographiques de Dakar Thiaroye. 
$q(y)$. La variance d'estimation ne dépend que de la distance inter-transect $\mathrm{d}$ et du covariogramme transitif 1D des biomasses par transect. Dans la formule, c'est le modèle de covariogramme qui est utilisé.

Dans la présente étude, les transects sont parallèles et orientés Est-Ouest. On considère que $\eta(y)$ est connue et non estimée car l'échantillonnage est continu. L'écho-intégration fournissant une valeur de densité moyenne par ESDU de longueur $a(a=$ 1 mille), $q(y)$ est calculée par sommation:

$$
q(y)=a \sum_{i=1}^{\prime \prime y} \bar{z}\left(x_{i} \cdot y\right)
$$

où $n_{y}$ est le nombre d'échantillons élémentaires (ESDU) sur le transect de latitude $y$ at $\bar{z}\left(x_{i}, y\right)$ est la densité moyenne dans l'ESDU d'indice $i$ sur le transect de latitude $\%$.

Le covariogramme transitif expérimental a été calculé comme suit:

$$
g^{*}(k d)=\sum_{j=1}^{n-k} q\left(y_{j}\right) q\left(y_{j}+k d\right)
$$

pour $k=0,1,2, \ldots n-1$, où $n$ est le nombre de transects.

Les modèles de covariogramme utilisés ont été l'effet de pépite (pep), le modèle triangle (tri) et le modèle sphérique (sph) dont les formules (Matheron, 1971) sont données dans le Tableau 2. L'effet de pépite caractérise une discontinuité dans la distribution spatiale (forte disparité entre valeurs très voisines). Lc modèle triangle décroît de façon linéaire et caractérise à 1D une distribution constante sur la zone d'étude (segment). La portée du modèle triangle est la taille de la zone (longucur du segment). Le modèle sphérique caraclérise une structuration (de type sphérique) dont la portée mesure la dimension moyenne.

La variance d'estimation pour un effet de pépite pur (: est égale à la distance inter-transect $d$ multiplićc par la valeur $c$ de la pépite. Comme les modèles triangles et sphériques ont une portée (distance à laquelle le modèle s'annule), la somme discrète et l'intégrale du covariogramme se réduisent à des sommations

Tableau 2. - Formules des modèles de covariogramme transitif utilisés. Le palier est noté c: et $r$ la portée.

\begin{tabular}{|c|c|}
\hline Modèle & Formule \\
\hline Eiffet de pé & $\begin{array}{l}g(0)=c \\
g\left(\left|f_{l}\right|\right)=0 \text { si }|h|>0\end{array}$ \\
\hline Triangle & $\begin{array}{l}g(|h|)=c(r-|h|) \text { si }|h|<r \\
g(|h|)=0 \text { si }|h| \geq r\end{array}$ \\
\hline Sphérique & $\begin{array}{l}g(|h|)=r\left(1-1,5|h| / r+(0.5)|h|^{3} / r^{3}\right) \text { si }|h|<r \\
g(|h|)=0, \mathrm{si}|h| \geq r\end{array}$ \\
\hline
\end{tabular}

Formulae of the transitive covariogram models used. finies. L’intégrale du covariogramme [deuxième terme dans la formule (1)] est $0,75 \mathrm{cr}$ pour le modelc sphérique et $r^{2}$ pour le modèle triangle. Le calcul du covariogramme expérimental, sa modélisation et le calcul de la formule (1) ont été effectués avec le logiciel EVA (Petitgas et Prampart, 1995).

\section{Application aux données}

I.es valcurs de biomasses par transect, valeurs $q(\eta)$. ont été calculées et leurs variations latitudinales ont été décrites. Ceci a conduit à regrouper les campagnes en 4 catégories. Pour chaque campagne, on a calculé le covariogramme transitif expérimental de $q(y) / \mathrm{Q}^{*}$ (covariogramme relatif). Le covariogramme relatif moyen pour chaque catćgorie a été ensuite calculé en prenant pour chaque distance la moyenne des covariogrammes des campagnes appartenant à cette catégorie. Les covariogrammes moyens par catégorie ont été ensuite modélisés et la variance d'estimation moyenne par catégorie a élé calculée.

\section{RÉSULTATS}

\section{Variation latitudinale des biomasses}

On a représenté pour chaque campagne, la contribution des transects à la biomasse totale estimée: $p(y)=\eta(y) / \mathrm{Q}^{*}$. Le profil latitudinal des pourcentages de biomasse se présente comme un processus stochastique à une dimension (Fig. 1). De grandes différences existent entre les profils des différentes campagnes. Toutefois, 4 catégories ont été reconnues.

Catégorie I: correspondant à l'année 1988. La biomasse est répartie de façon très inhomogène et aucune structuration n'est visible. La biomasse est concentrée sur un nombre très faible de radiales (distribution aléatoire des $p(y)$ et forte variance des $p(y))$.

Catégoric II: correspondant à l'annéc 1984. La biomasse est répartie de façon homogène sur le profil mais aucune structuration n'est visible (distribution aléatoire de $p(y)$ et variance plus faible).

Catégorie III: concernant les années 1985, 1986, 1987, 1992 et 1993. La biomasse est répartie de façon homogène sur le profil et une petite structuration est visible. Des transects proches ont des valeurs de $p(y)$ proches (répartition structurée de $\nu(y)$ avec une petite portée et variance faible).

Catégorie IV : l'année 1989. La biomasse est répartie sur le profil de façon homogène, une structuration est visible et sa portée est plus grande que précédemment 
$1984 \mathrm{Q}=288959$ tonnes

$1985 a=744051$ tonnes

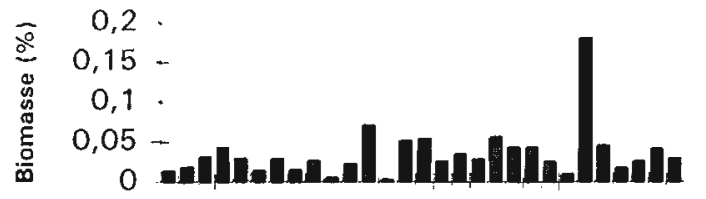

$\begin{array}{llllllllll}1 & 4 & 7 & 10 & 13 & 16 & 19 & 22 & 25 & 28\end{array}$

Transects

$1986 \mathrm{Q}=435960$ tonnes

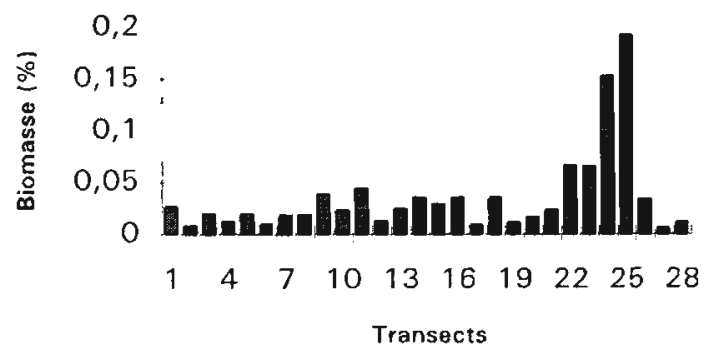

$1988 Q=463333$ tonnes

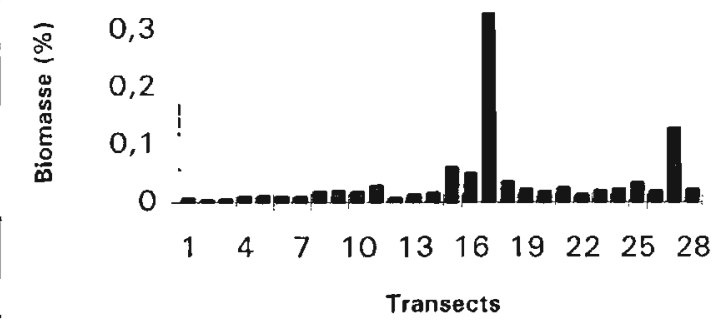

$1992 \mathrm{Q}=368658$ tonnes

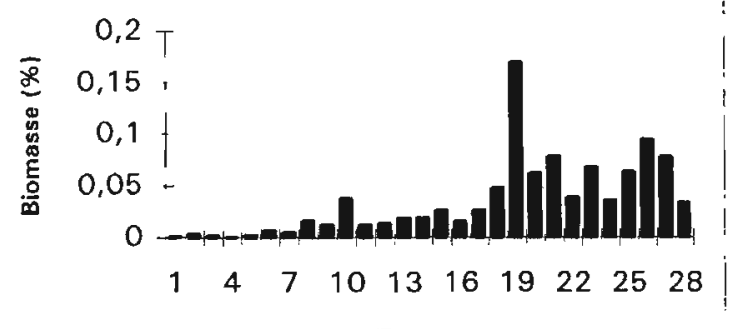

Transects

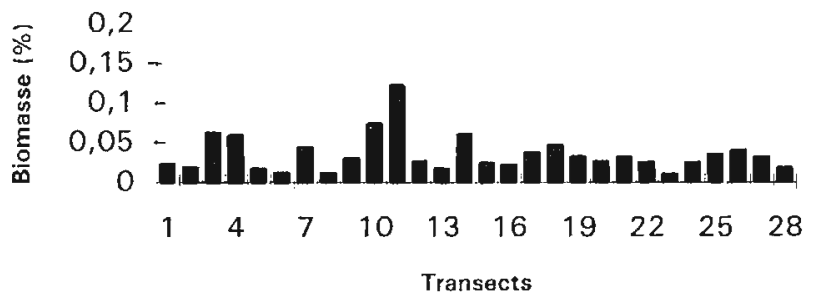

$1987 Q=338518$ tonnes

0,2

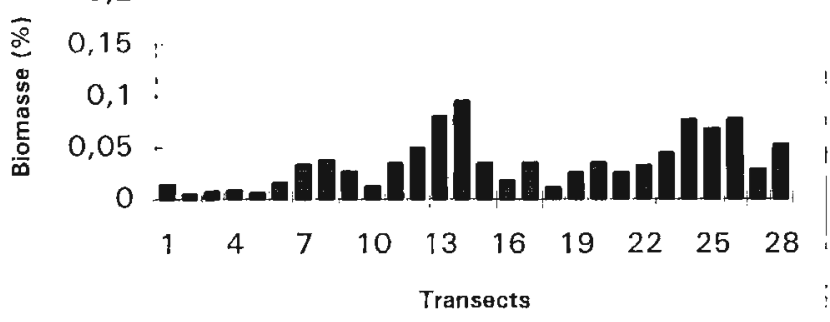

$1989 Q=86374$ tonnes

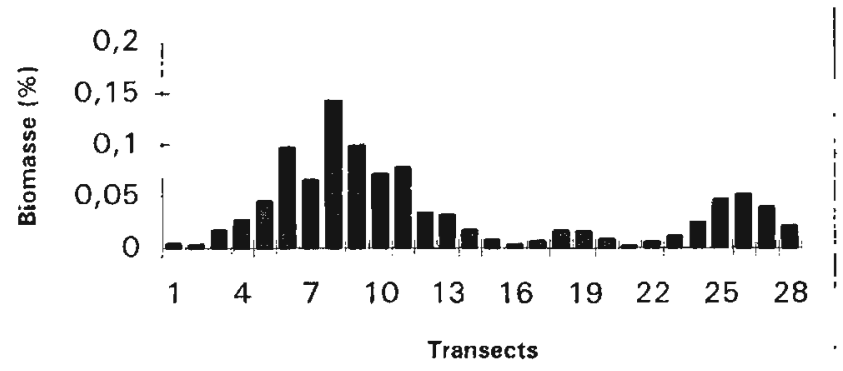

$1993 Q=652258$ tonnes

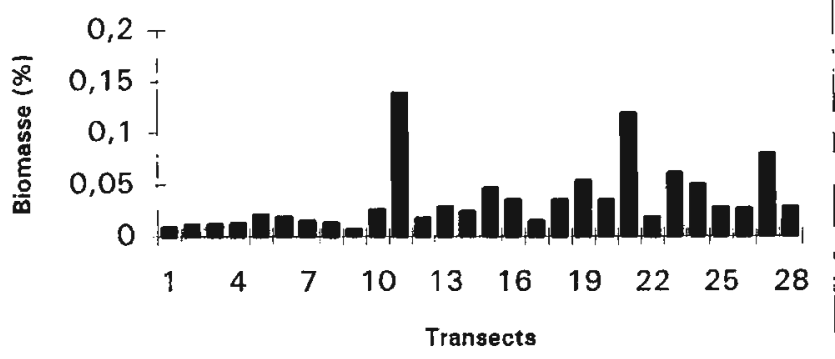

Figure 1. - Distribution à une dimension de la biomasse par transect pour chaque campagne acoustique. Catégorie I: 1988, Catégorie II: 1984 , Catégorie 1II: 1985-1986-1987-1992-1993, Catégorie IV: 1989.

J-dimensional spatial distribution of biomass per transect for each survey. Caregory I: 1988, Categon 11: 1984, Category 111: 1985-19861987-1992-1993, Category IV: 1989. 
(répartition structurée des $p(y)$ avec une portée grande et variance faible). La biomasse estimée lors de cette campagne est beaucoup plus faible que pour toutc autre campagne de la séric (Tablcau 1).

Sur les 8 campagnes considérées, 5 appartiennent à la catégorie III. Les transects riches [ $\eta(y)$ fort] n'ont pas toujours la même localisation. La valeur de la biomasse estimée dépend des quelques transects riches. Ces fortes valeurs sont dues à la présence de quelques fortes valeurs de densité sur les transects.

\section{Covariogrammes transitifs}

Les covariogrammes transitifs expérimentaux moyens ont été représentés pour chaque catégoric et leurs modélisations sans effet de pépite (Fig. 2). Les caractéristiques des profils décrites précédemment se retrouvent sur les covariogrammes transitifs. La forte variance de l'annćc 1988 se traduit par une valcur du covariogramme à l'origine très forte $(0,7)$. Les covariogrammes des autres catégories ont des valeurs à l'origine comparables (proches de 0,35 ). L'aspect fortement aléatoire des catégories I et II se marque par une chute rapide du covariogramme (grande disparité entre transects voisins) dès la première distance puis par une lente décroissance linéaire compatible avec les modèles triangle ou sphérique. En revanche, les covariogrammes transitifs des catégories III et IV montrent une structuration compatible avec un modèle sphérique. Pour les catégories I et II surtout, la présence d'un effel de pépite reste indéterminćc. L'effet de pépite (Petitgas, 1993) devrait être théoriquement très faible à 1D car la sommation sur les transects tend à la filtrer. Toutefois, on a effectué 2 modélisations pour chaque campagne, avec et sans effet de pépite. Les modèles utilisés et les valeurs de leurs paramètres sont donnés dans le Tableau 3.

\section{Variances d'estimation}

Les modèles précédents sont maintenant utilisés pour estimer la variance d'estimation selon la formule (1). Pour chaque catégorie, on a deux valeurs de variance, une pour le modèle sans pépite et une autre pour le modèle avec pépite. Puis on a calculé l'erreur relative d'estimation, $\sigma_{Q} / \mathrm{Q}^{*}$, pour chaque campagne et dans chaque catégorie, on a retenu la valeur moyenne de ce rapport. Les erreurs relatives d'estimation $\left(\sigma_{Q_{2}} / Q^{*}\right)$ sont consignées dans le Tableau 4. La prise en comple d'un effet de pépite a une grande influence sur l'erreur relative estimée. L'errcur rclative d'estimation se trouve dans une fourchette entre 5 et $31 \%$. Cela dépend, d'une part des différences de structuration selon les différentes années, el d'autre part de la prise en compte ou non d'un effet de pépite.

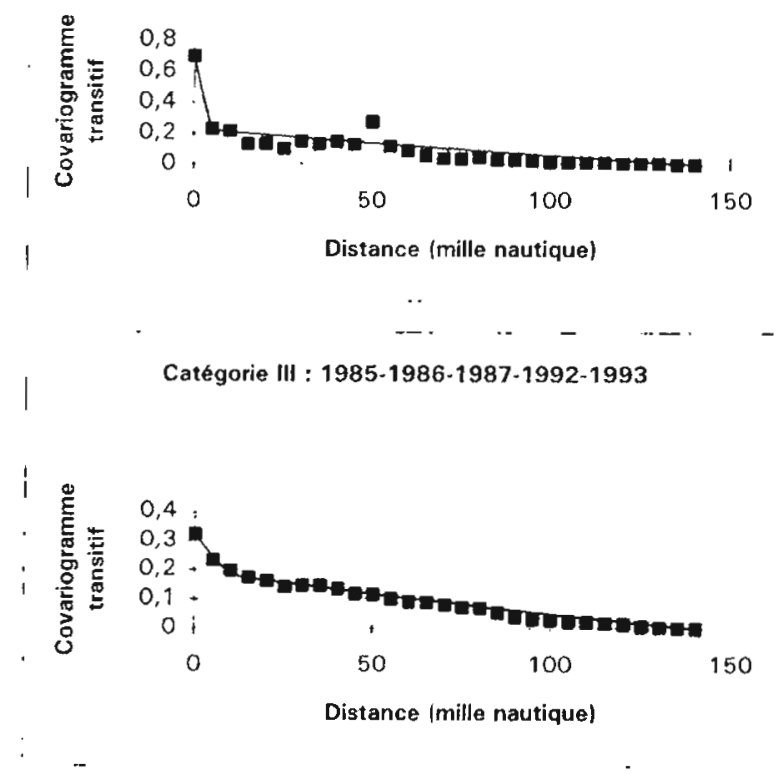

Catégorie II : 1984
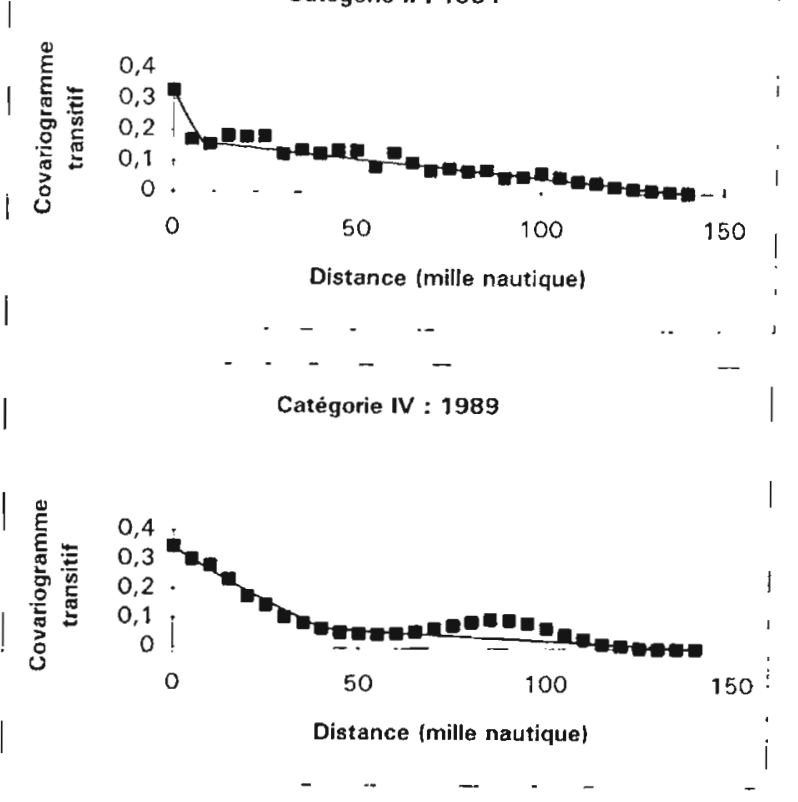

Figure 2. - Covariogrammes transitifs moyens pour chaque catégorie de structure spatiale à une dimension.

Average transitive covariogram for each category of I-dimensional spatial stracture. 
Tableau 3. - Modèles de covariogramme transitif utilisés pour chaque catégorie de structure. Les formules des modèles sont données dans le tableau 2. $\operatorname{sph}(c, r)=$ sphérique de palier $c$ et portée $r$. tri $(r, r)=$ triangle de palier $c$ et portéc $r$, pep $(c)=$ pépite de constante $c$.

Models of transitive covariogram used for each category of ID spatial structure. The formulas for these models are given in table 2. sph $(c, r)$ $=$ spherical with sill $c$ and range $r$. trif $c, r)=$ triangular with sill $c$. and range $r$. pep $(c)=$ nugget effect of value $c$.

\begin{tabular}{lll}
\hline $\begin{array}{l}\text { Type de } \\
\text { catégorie }\end{array}$ & $\begin{array}{l}\text { Modélisation } \\
\text { sans pépite }\end{array}$ & $\begin{array}{l}\text { Modélisation } \\
\text { avec pépite }\end{array}$ \\
\hline I & $\begin{array}{l}\operatorname{sph}(0.47,5) \\
+\operatorname{sph}(0.23,140)\end{array}$ & $\begin{array}{l}\operatorname{pep}(0.47) \\
+\operatorname{sph}(0.23,140)\end{array}$ \\
\hline II & $\begin{array}{l}\operatorname{sph}(0.16,8) \\
+\operatorname{tri}(0.17,140)\end{array}$ & $\begin{array}{l}\operatorname{pep}(0.16) \\
+\operatorname{tri}(0.17,140)\end{array}$ \\
\hline III & $\begin{array}{ll}\operatorname{sph}(0.122,10) \\
+\operatorname{sph}(0.2,140)\end{array}$ & $\begin{array}{l}\operatorname{pep}(0.032)+\operatorname{sph}(0.09,10) \\
+\operatorname{sph}(0.2,140)\end{array}$ \\
\hline IV & $\begin{array}{l}\operatorname{sph}(0.26,45) \\
+\operatorname{sph}(0.1,140)\end{array}$ & $\begin{array}{l}\operatorname{pep}(0.02)+\operatorname{sph}(0.24,45) \\
+\operatorname{sph}(0.1,140)\end{array}$ \\
\hline
\end{tabular}

Tableau 4. - Erreurs relatives d'estimation $\left(\sigma_{Q} / \mathrm{Q}^{*}\right)$ pour chaque catégorie de structure, avec et sans pépite.

Relative estimation errors for each category of structure, with and without nugget effect.

\begin{tabular}{llll}
\hline $\begin{array}{l}\text { Type de } \\
\text { catégorie }\end{array}$ & $\begin{array}{l}\text { Noms } \\
\text { des campagnes }\end{array}$ & $\begin{array}{l}\text { Erreur relative } \\
\text { sans pépite \% }\end{array}$ & $\begin{array}{l}\text { Erreur relative } \\
\text { avec pepitc \% }\end{array}$ \\
\hline I & Echosar 14, 1988 & 15,4 & 30,7 \\
\hline II & Echosar 06, 1984 & 7,5 & 17,9 \\
\hline III & $\begin{array}{l}\text { Echosar 08, 1985 } \\
\text { Echosar 10, 1986 } \\
\text { Echosar 12, 1987 }\end{array}$ & 5,8 & \\
& $\begin{array}{l}\text { Echosar 16, 1992 } \\
\text { Echosar 17, 1993 }\end{array}$ & 9,5 \\
\hline IV & Echosar 15, 1989 & 4,0 & 7,4 \\
\hline
\end{tabular}

\section{DISCUSSION ET CONCLUSION}

Sur la côte Sud du Sénégal où les campagnes d'évaluation acoustique ont été réalisées suivant un réseau de transects parallèles et équidistants, la méthode géostatistique transitive à une dimension offre une méthode adaptée et simple pour l'estimation de la précision de l'échantillonnage. La variance d'estimation dépend du modèle de structure spatiale utilisé (autocorrélation de la biomasse par transect). Elle est conditionnée par nos connaissances ou par les hypothèses sur le phénomène. D'une part, la structuration spatiale varie selon les campagnes de façon importante, d'autre part, la modélisation de la variation, à petite échelle, de la biomasse par transect reste indéterminée car on ne sait pas s'il est judicieux de prendre en compte une pépite à l'origine du covariogramme. L'erreur relative maximale a été estimée à presque $31 \%$.

Des structurations-types, pour le profil latitudinal de la biomasse par transect, ont été reconnues. Certaines campagnes présentent des structurations particulic̀rcs. Seule la catégorie III (structuration de petite portée et faible variance) est bien représentée. Cette structuration est donc la plus probable. Les différences de biomasses entre les campagnes de la catégorie III sont similaires aux différences entre catégories. On peut done s'interroger si les structurations-types sont réellement liées à la biomasse. D'autres facteurs entrent vraisemblablement en jeu, tels que l'environnement hydrologique (histoire de l'upwelling) ou le régime migratoire des principales espèces pélagiques dans la zone d'étude (Boely et al., 1982). Toutefois, la biomasse très faible de 1989 est marquée par une structuration très différente. Le lien entre structuration et biomasse ne semble pas être un simple lien de densité-dépendance.

Dans l'application de la méthode, la prise en compte d'un effet de pépite à $1 \mathrm{D}$ sur les valeurs cumulées par transect semble dictée par les données mais pose un problème méthodologique. L'effet de pépite à 2 D caractérise le fait que deux ESDUs proches ont des valeurs très différentes. Cette modélisation permet de décrire la variation très locale à $2 \mathrm{D}$ car physiquement, l'agrégation en bancs engendre une discontinuité dans les valeurs de densité à petite échelle spatiale. Cependant une telle pépite à 2D, par la sommation le long des radiales est en théorie, sinon filtrée, réduite à $1 \mathrm{D}$ sur les valeurs cumulées par transect (Petitgas, 1993). Si une pépite est présente à $1 \mathrm{D}$ expérimentalement, son origine n'est pas à rechercher dans la discontinuité des densités à 2D due à l'agrégation en bancs. Si nous acceptons la réalité physique d'une pépite à $1 D$ pour la biomasse par transect, nous devons pouvoir lui attribuer une interprétation biologique. Nous pensons qu'elle pourrait traduire une variation temporelle. Sous cette appellation sont regroupées différentes sources de variations, les variations jour-nuit, les mouvements et autres changements. Le long du transect, pour les petites distances au moins, l'échantillonnage est spatial dans la mesure où, le bateau allant à 9 nœuds, on échantillonne la distribution spatiale telle qu'elle est dans l'instantané. Entre les transects en revanche, les variations de la biomasse par transect contiennent une certaine part de variation temporelle en plus de la variation spatiale latitudinale. Dans cette étude, la valeur même de la pépite ID que l'on pourrait utiliser n'est pas connue avec précision. Des répétitions de transects lors de l'échantillonnage pourraient permettre d'accéder à la modélisation d'une pépite à $1 \mathrm{D}$ et ainsi permettre une meilleure prise en compte de la variabilité temporelle dans l'estimation de la précision sur l'évaluation de la biomasse.

Les deux approches suivantes pour calculer la variance d'estimation, à 2 dimensions dans le plan longitude - latitude avec le variogramme des densités 
par ESDU ou à I dimension avec les biomasses par transect et leur covariogramme transitif sans clfet de pépite, sont équivalentes en l'absence de variations temporelles (Matheron 1971, Petitgas 1993). La validation de la méthode ID ne peut pas venir de la comparaison entre l'étude 2D et 1D. Williamson et Traynor (1996) ont cherché une validation en comparant la variance estiméc par la méthode 1D à la valeur expérimentale de variance entre échantillonnages répétés sur une même zone. Sous réserve que la biomasse reste constante sur zone et que la variance expérimentale soit estiméc avec précision, la méthode $1 \mathrm{D}$ sans effet de pépite était inférieure à la valcur expérimentale. On peut s'interroger sur l'utilité de rajouter un effet de pépite à une dimension.

Alors que les données contiennent une variabilité spatio-temporelle, le traitement uniquement spatial des campagnes d'évaluation conduit à prédire une variance d'estimation nulle pour un échantillonnage exhaustif de l'espace. Cette prédiction n'est pas réaliste car l'échantillonnage est par nature spatio-temporel à cause de l'interaction entre espace et temps au cours du trajet du bateau. Des transects répétés lors des campagnes d'écho-intégration et la valeur de la pépite à une dimension pourraient permettre une caratćrisation simple de la variabilité temporelle à l'échelle des transects.

\section{Remerciements}

Nous remercions M. J.J. Levenez qui fut co-responsable des campagnes Echosar de 1984 à 1994. Nous remercions l'équipage du N/O "Louis Sauger " ainsi que MM. I. Sow, M. Sylla, $\wedge$. Sarre et I. Sane, personnels techniques du CRODT, sáns lesquels les données n'auraient pu être récoltécs ni mises en forme.

\section{RÉFÉRENCES}

Boely T., J. Chabannc, P. Fréon, B. Stequert 1982. Cycle sexuel et migrations de Sardinella aurita sur le platcau continental ouest africain des îles Bissagos à la Mauritanie. Rapp. P-V. Réun. Cons. Int. Explor. Mer $180,350-355$.

Cochran W. 1977. Sampling techniques. John Wiley and Sons, New York.

Johanesson K., R. Mitson 1984. Fisheries acoustics: a practical manual for aquatic biomass estimation. FAO Fish. Tech. Pap. 240.

Jolly G., I. Hampton 1990. A stratified random transect design for acoustic surveys of fish stocks. Can. J. Fish. Aquat. Sci. 47, 1282-1291.

Levenez J.J. 1990. Mesures de l'index de rélexion acouslique (TS) de quelques poissons pélagiques tropicaux par la méthode de la cagc. Archive Cent. Rech. Océanogr. Dakar-Thiaroyc, CRODT n 117.

Petitgas P. 1993. Geostatislies for fish stock assessments: a review and an acoustic application. ICES J. Mar. Sci. 50, 285-298
Petitgas P., A. Prampart 1995. EVA: Estimation Variance, logiciel de Géostatistique pour l'analyse structurale et les calculs de variance. Editions de l'ORSTOM, logOrstom, Paris.

Matheron G. 197I. La théorie des variahles régionalisćes et son application. Cah. Morphol. Math. 5, École des Mines de Paris, Fontainehleau.

Samba A. 1994. Présentation sommaire des différentes pêcheries sénégalaises. In: L'évaluation des ressources exploitables par la pêche artisanale sénégalaise. M. BarryGérard, T. Diouf, A. Fonteneau eds. ORSTOM-Editions, Paris, $1-10$.

Simmonds E., N. Williamson, F. Gerlotto, A. Aglen 1992. Acoustic survey design and analysis procedure: a comprchensive review of current practice. ICES Cooperative Res. Rep. 187.

Williamson N., J. Traynor 1996. Application of a onedimensional geostatistical procedure to fisheries acoustic surveys of Alaskan pollock. ICES J. Mar. Sri. 53, 423-428. 